\title{
PARTÍCULAS DE ORO EN PLACERES LATERALES MARINOS (NORESTE DE CUBA): MORFOLOGÍA, TEXTURA Y COMPOSICIÓN
}

\author{
Alberto Vila-Sánchez ${ }^{1}$, Roberto Díaz-Martínez ${ }^{1}$, \\ Joaquín Proenza-Fernández², Joan Carles Melgarejo ${ }^{2}$ \\ ${ }^{1}$ Departamento de Geología, Instituto Superior Minero Metalúrgico de Moa. \\ Las Coloradas, s/n, 83320, Moa, Holguín, Cuba. avila@ismm.edu.cu \\ 2 Departament de Cristalografia, Mineralogia i Dipòsits Minerals. Facultat de \\ Geologia, Universitat de Barcelona. Martí i Franquès, s/n 08028 Barcelona, España
}

(Recibido 9/12/03; aceptado 3/2/04)

\begin{abstract}
The main morphologic and textural features, and the geochemistry of the particles of gold from marine placers from Mejías and Jiguaní beaches (Mayarí-Moa-Baracoa Ophiolitic Massif, northeast of Cuba) are described. This work aims to discuss the formation mechanisms (chemical accretion vs. mechanical accretion) and the primary sources of the gold. The particles of Au from both deposits are very fine, showing predominantly tabular shapes and occasionaly thin sheets or flakes. The particles exhibit complex internal textures, with concave and convex boundaries, as well as rotten grains. They are composed of subgrains of different composition. Several subgrains display electrum, native gold and mercurial gold, and alloys of $\mathrm{Au}-\mathrm{Cu}$ (tetra-auricupride) in the placer Jiguaní. Usually, these subgrains have alteration rims, consisting in a gold enrichment at the expense of silver.

The occurrence of subgrains of different composition within one particle, cannot be explained by a simple process of chemical accretion. On the other hand, these textures suggest that the particles originated from a process of mechanical accretion of gold particles, favoured by the malleability of this metal. The surfaces of separation of the subgrains have acted as planes of structural weakness, favouring the chemical alteration of the particles. Observed textures indicate a multiepisodic process of formation.

The chemical composition of the investigated auriferous particles reveals electrum ( $\mathrm{Au}-\mathrm{Ag}$ ), native gold ( $\mathrm{Au})$ and mercurial gold (Au-Hg). In both placers the gold can be related to the presence of hydrothermal gold-rich veins and breccias in the region. However, contribution of other possible primary sources, like ophiolitic rocks, listvenites and laterites, cannot be discarded.
\end{abstract}

Keywords: Particles of gold, morphologic, textural, and chemical composition, lateral marine placers.

RESUMEN: En este trabajo se describen los principales rasgos morfológicos, texturales y composicionales de las partículas de oro presentes en los placeres marinos costeros de las playas Mejías y Jiguaní, ubicados en la porción noreste de Cuba (Macizo Ofiolítico Mayarí-Moa-Baracoa). A partir de este estudio se discute sobre los posibles mecanismos de formación (acreción química vs. acreción mecánica) y las fuentes primarias de las partículas de oro. 


\begin{abstract}
Las partículas de Au estudiadas en ambos depósitos son muy finas, mostrando formas tabulares predominantes, en ocasiones aparecen muy aplastadas, formando especies de laminillas u hojuelas. Por medio de las imágenes de electrones retrodispersados se observan texturas internas muy complejas, así como cariado de los granos. Las partículas están compuestas por subgranos de composición diferente, correspondientes a aleaciones naturales de electrum, oro nativo y mercurial en ambos placeres y aleaciones de $\mathrm{Au}-\mathrm{Cu}$ (tetra-auricuprido) en el placer Jiguaní. Los subgranos frecuentemente se encuentran alterados en sus bordes, que consiste de un enriquecimiento en oro con relación a la plata. Las texturas observadas no pueden ser explicadas mediante un proceso simple de acreción química. En cambio, estas texturas sugieren un origen de las partículas a partir de un proceso de acreción mecánica de las partículas de oro, favorecido por la maleabilidad de este metal. Las superficies de separación han actuado como superficies de debilidad estructural, favoreciendo la alteración química de las partículas. Las texturas observadas indican un proceso multiepisódico de formación de las partículas.

La composición química de las partículas auríferas investigadas corresponde con aleaciones naturales de electrum $(\mathrm{Au}-\mathrm{Ag})$, oro nativo $(\mathrm{Au})$ y oro mercurial $(\mathrm{Au}-\mathrm{Hg})$ en ambos placeres, la que puede estar vinculada principalmente con la presencia de filones y brechas hidrotermales enriquecidas en oro descritas en la región de estudio. Sin embargo, no se puede descartar el aporte de otras posibles fuentes primarias, ya que en la región existen diversos metalotectos para oro: rocas ofiolíticas, listvenitas, lateritas.
\end{abstract}

Palabras clave: Partículas de oro, morfología, textura y composición química, placeres marinos laterales.

\section{INTRODUCCIÓN}

Las acumulaciones secundarias superficiales marino-costeras de la región Sagua-Moa se caracterizan por la presencia de abundantes granos de oro (Kulaskov et al., 1990; Díaz-Martínez, 1995; Vila, 1999). Los placeres laterales marinos de las playas Mejías y Jiguaní (Fig. 1), constituyen los primeros ejemplos de placeres marinos descubiertos en el noreste de Cuba, los cuáles presentan importantes concentraciones de oro en asociación a diversos minerales pesados (Díaz-Martínez, 1995).

Las fuentes primarias de estas partículas pudieran estar relacionadas con los complejos ofiolíticos, listvenitas, depósitos de sulfuros masivos mesozoicos y filones epitermales presentes en la región de estudio. Además, cabe añadir los procesos intensos de laterización presentes en la región, cuya influencia sobre cualquier tipo de mineralización debe ser analizada.

En este trabajo se define la morfología, textura y la geoquímica mineral del oro en estos depósitos de placeres, con el objetivo de determinar la composición de las posibles áreas fuentes.

El análisis combinado de las características morfotexturales y composicionales de las partículas de oro investigadas proporcionan información, sobre la composición y posición relativa de las fuentes portadoras de la mineralización aurífera respecto a los depósitos estudiados y sobre los mecanismos de formación (acreción química y/o mecánica) de los granos de oro.

Pocos ejemplos de placeres de playa enriquecidos en oro son citados en la literatura mundial. Entre los más importantes están los de la costa de Alaska y de California (Sawkins, 1986), que se han formado a partir de la meteorización de vetas de oro orogénico. En California las tres cuartas partes del oro de Mother Lode se ha recuperado de los placeres, en especial en los de tipo playa.

\section{CONTEXTO GEOLÓGICO REGIONAL}

La región de estudio, situada en el noreste de Cuba Oriental (Fig.1) se caracteriza por el predominio de las rocas pertenecientes a la Faja Ofiolítica Mayarí-Baracoa (Fig. 2; Iturralde-Vinent, 1996a; Proenza et al., 1999a,b), la cuál ocupa una área aproximada de $1500 \mathrm{~km}^{2}$. En esta faja ofiolítica se han descrito tanto niveles mantélicos (Jurásico-Cretácico Temprano) como corticales (Hauteriviense-Campaniense) (Iturralde-Vinent, 1996a). Se pueden identificar todos los niveles de un corte ofiolítico ideal, aunque desmembrado tectónicamente (Proenza et al., 1998; Proenza et al., 1999a; 1999b): peridotitas con texturas de tectonitas, rocas representativas de la Moho Transition Zone, cumulados máficos, diques de diabasas y niveles efusivos sedimentarios. Las secuencias mantélicas y los cumulados de las 


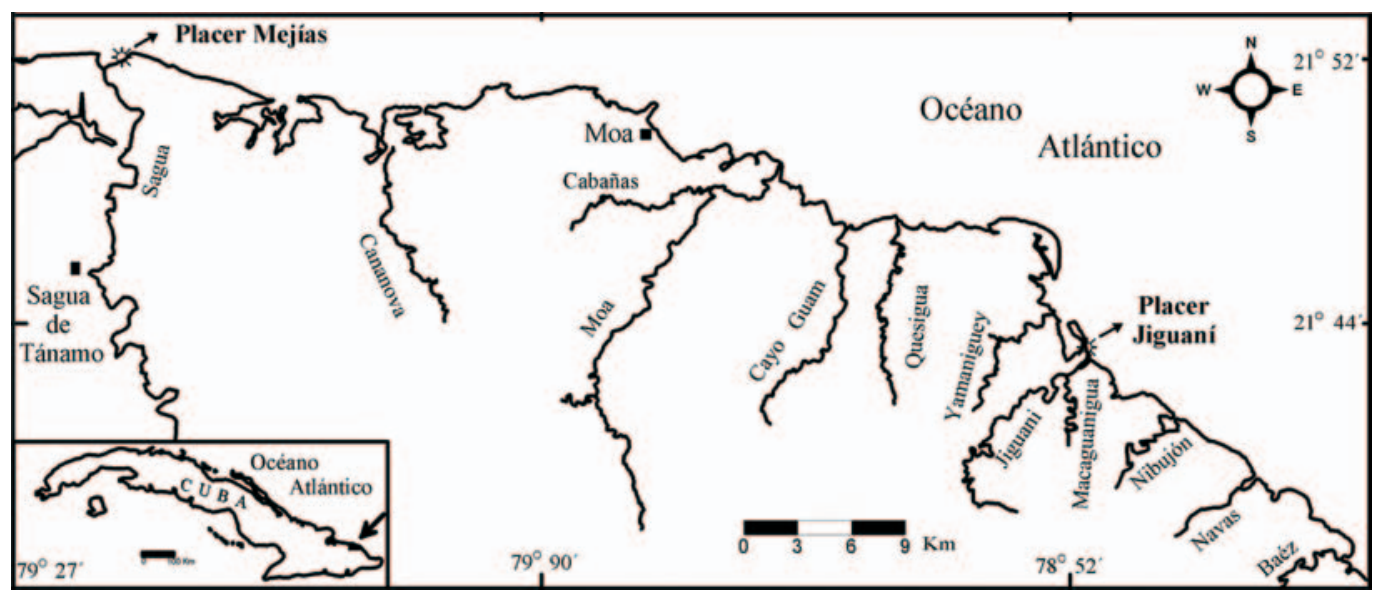

Fig. 1: Mapa de ubicación de la región de estudio, noreste de Cuba oriental.

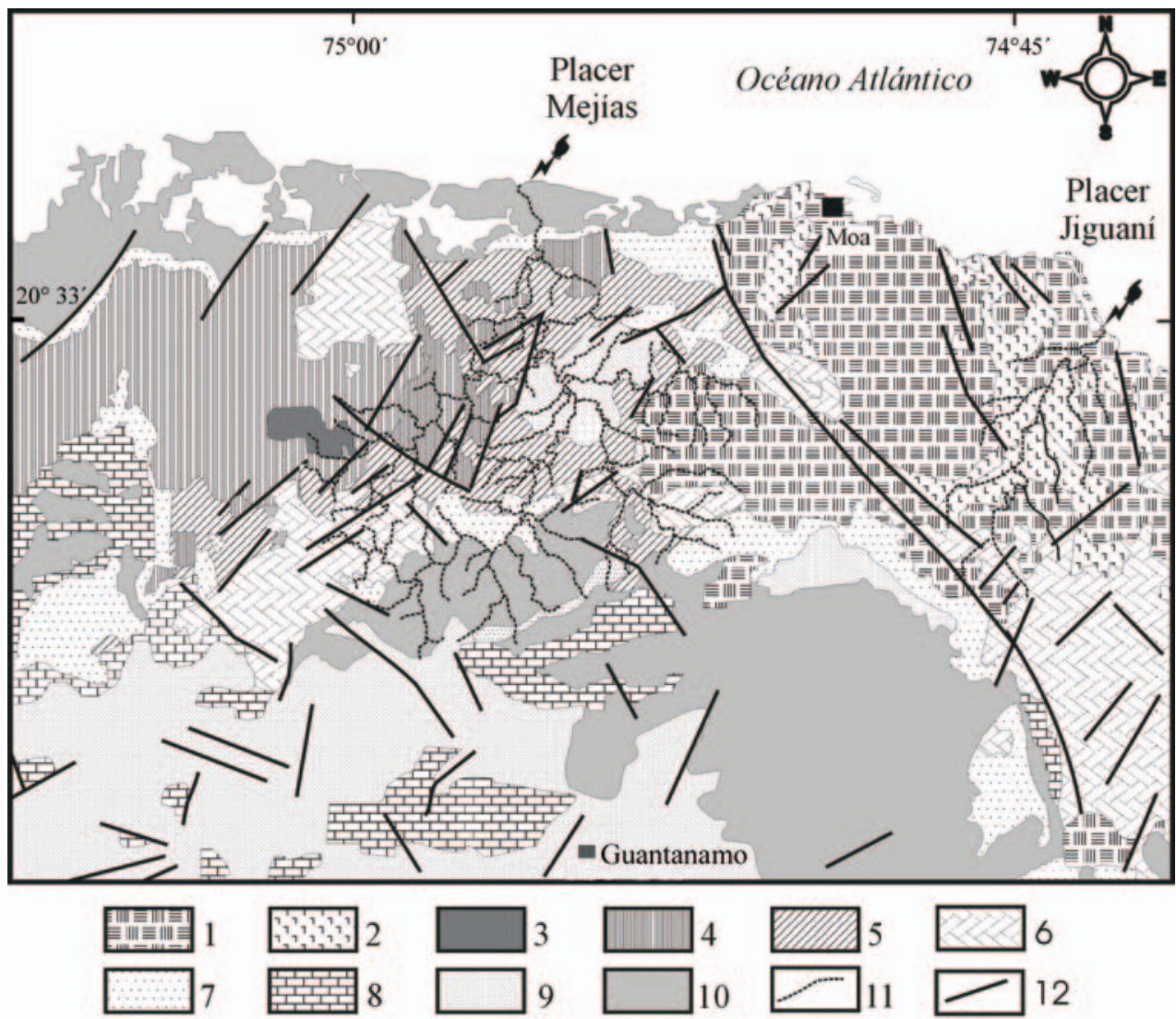

Fig. 2: Mapa geológico de la región estudiada: 1) Macizo ofíolítico Moa-Baracoa (constituido por peridotitas, principalmente harzburgitas); 2) Gabros del complejo de acumulados máficos; 3) Complejo metamórfico “La Corea” (anfibolitas, plagiogneis); 4) Macizo ofíolítico Mayarí-Cristal (constituido por peridotitas, principalmente harzburgitas y dunitas); 5) Materiales sedimentarios detríticos asociados a las cuencas transportadas (piggy back) del Campaniense tardío al Daniense ; 6) Rocas volcánicas y volcanosedimentarias del arco de islas volcánico del Cretácico; 7) Rocas volcánicas y volcanosedimentarias del arco de islas volcánico del Paleógeno. Cuencas transportadas (piggy back) del Eoceno Medio al Superior (Oligoceno?); 8) Formaciones carbonatadas; 9) Molasa flyschoide. Cobertura Neógena-Cuaternaria; 10) Calizas, gravas, arenas, arcillas; 11) Red Fluvial; 12) Fallas. 
ofiolitas Mayarí-Baracoa se han datado como Jurásico-Cretácico Temprano y las rocas volcano-sedimentarias como Hauteriviense (?)-Campaniense (?) (Iturralde-Vinent, 1996a).

Los gabros forman grandes cuerpos incluidos en el complejo ultramáfico. La dimensión de los cuerpos oscila entre 1 y $3 \mathrm{~km}$ de ancho y 10 a $15 \mathrm{~km}$ de longitud. El contacto entre los niveles ultramáficos y de gabros es generalmente tectónico. Muchas veces los gabros están cubiertos por mantos de rocas ultramáficas (Fonseca et al., 1985), aunque otros autores (Andó et al., 1989) plantean que en algunos sectores el contacto es transicional.

Los niveles volcánicos y volcánicos-sedimentarios están en contacto tectónico con los demás niveles de la secuencia ofiolítica. Las rocas volcánicas constituyen la Formación Quiviján, la cual tiene un espesor superior a los $500 \mathrm{~m}$, y una edad probablemente pre-Albiense. Está constituida por basaltos amigdaloidales y porfiricos (algunas veces con estructura de almohadilla) con intercalaciones de hialoclastitas, tobas, capas de cherts y calizas (Quintas, 1989; Iturralde-Vinent, 1996a; Kerr et al., 1999). Las rocas basálticas tienen una afinidad geoquímica de toleitas de arcos de islas, y han sido interpretadas como generadas en una cuenca tras-arco.

Bajo las ofiolitas yacen, a través de una falla subhorizontal, rocas volcano-sedimentarias del arco de islas Cretácico. Aunque en otras regiones los cuerpos ofiolíticos aparecen cubiertos por materiales volcano-sedimentarios pertenecientes al arco de islas del Paleógeno (Formación Sabaneta) y por secuencias terrígenas-carbonatadas más jóvenes (Quintas, 1989; Iturralde-Vinent, 1996b). Los principales afloramientos de la Faja Ofiolítica Mayarí-Baracoa están representados por los macizos Mayarí-Cristal y Moa-Baracoa (Proenza et al., 1998); Proenza et al.,1999a; 1999b).

Al sur del Macizo Mayarí-Cristal, se localiza el "melange la Corea" (Fig. 2), una zona metamórfica de unos $25 \mathrm{~km}^{2}$ (Adamovich \& Chejovich, 1964; Nagy et al., 1983; Millán, 1996). Esta zona se compone de diferentes bloques separados por una matriz de serpentinítas. Predominan las rocas metamórficas de alta presión, así como metabasitas de baja presión de origen ofiolítico (Millán, 1996). Las metamorfitas de alta presión son anfibolitas granatíferas y bloques aislados de esquistos glaucofánicos; además, existen esquistos verdes, esquistos tremolíticos, actinolíticos, diques de pegmatitas y granitoides masivos.

En la región de estudio también afloran rocas volcánicas y volcano-sedimentarias de edad cretácica, las cuáles están representadas por la Formación Santo Domingo, que está compuesta por tobas y tufitas con cuerpos de lavas y calizas intercalados (Proenza \& Carralero, 1994). Los materiales de la Formación Santo Domingo se encuentran imbricados tectónicamente con las ofiolitas de la Faja Mayarí-Baracoa. Muchas veces los contactos coinciden con zonas que presentan una mezcla de bloques de volcanitas pertenecientes al arco y de ofiolitas. La actividad volcánica se extendió desde el Aptiense al Campaniense Medio (Iturralde-Vinent, 1994) y fue el resultado de una subducción intraoceánica.

Los materiales asociados a las cuencas transportadas del Campaniense Tardío-Daniense se componen de las formaciones Micara y $\mathrm{La}$ Picota. Dentro de estas formaciones se encuentran secuencias típicamente olistostrómicas y con características de melange (Formación La Picota y parte de la Formación Micara), las cuales están compuestas por fragmentos y bloques procedentes de la secuencia ofiolítica y de las rocas volcánicas Cretácicas. En consecuencia, estas unidades litoestratigráficas constituyen un registro temporal del emplazamiento tectónico (obducción) de las ofiolitas.

Las rocas volcánicas y volcano-sedimentarias de edad Paleoceno (Thanetiense)-Eoceno Medio Inferior están representadas por la Formación Sabaneta, la cuál ha sido depositada en una cuenca tras-arco (Iturralde-Vinent, 1996b; García-Torres et al., 1996). La unidad se compone de rocas epiclásticas (tobas, tufitas) (Iturralde-Vinent, 1976, 1996b; Proenza \& Carralero, 1994; Quintas, 1996, García-Torres et al., 1996). Las rocas pertenecientes al arco de islas volcánico del Paleógeno yacen sobre los materiales deformados del arco Cretácico, las ofiolitas y las cuencas de piggy back del Campaniense Tardío-Daniense.

Las series asociadas a las cuencas transportadas del Eoceno Medio al Oligoceno están 
constituidas por las formaciones terrígenas carbonatadas (Quintas, 1989; Quintas \& Blanco, 1993; Proenza \& Carralero, 1994): a) la Formación Charco Redondo (calizas microcristalinas, calizas margosas); b) Formación San Luis (areniscas y facies terrígenas carbonatadas hacia el norte); y Formación Maquey (margas, areniscas calcáreas, calizas organógeno-detríticas).

\section{MATERIALES Y MÉTODOS}

Se realizó un muestreo sistemático de los sedimentos marinos costeros de los placeres Mejías y Jiguaní (Cuadro 1), mediante el método de los concentrados pesados, conocido en Cuba como Método de Jagua, el cual ha sido ampliamente utilizado en la región de estudio por geólogos cubanos (ej: Díaz-Martínez, 1996; Díaz-Martínez et al., 1998). La metodología adoptada consistió en lavar las arenas, concentrarlas y rectificarlas. Posteriormente se realizó la separación granulométrica, magnética, electromagnética y densimétrica. Finalmente, con ayuda del binocular se obtuvo una muestra monomineral de 32 granos de oro en el placer Mejías y 28 partículas en los depósitos marinos costeros del placer Jiguaní, para un total de 60 partículas investigadas y se realizó una descripción morfo-textural así como las mediciones (largo, ancho y grosor) de los granos de oro.

Cuadro 1

Número de muestras recolectadas y cantidad de granos de oro extraídos en los Placeres marinos ubicados en la porción noreste de Cuba

\begin{tabular}{lccc}
\hline $\begin{array}{l}\text { Placeres } \\
\text { marinos }\end{array}$ & $\begin{array}{c}\text { Muestras } \\
\text { recolectadas }\end{array}$ & $\begin{array}{c}\text { Granos de oro } \\
\text { extraídos }\end{array}$ & $\begin{array}{c}\text { Métodos } \\
\text { empleados }\end{array}$ \\
\hline $\begin{array}{l}\text { Playa } \\
\text { Mejías }\end{array}$ & 8 & 32 & $\begin{array}{c}\text { Concentrados } \\
\text { pesados }\end{array}$ \\
$\begin{array}{l}\text { Playa } \\
\text { Jiguaní }\end{array}$ & 4 & 28 & \\
\hline
\end{tabular}

Además, se determinaron parámetros morfométricos tales como: diámetro equivalente (Deq) e índice de aplastamiento (IA), también conocido como factor de forma Corey (Giusti, 1986). El diámetro equivalente se determinó mediante la expresión:

$$
D_{e q}=\sqrt[3]{\frac{6}{\pi}} \cdot \mathrm{a} \cdot \mathrm{b} \cdot \mathrm{c}
$$

Donde:

Deq $=$ Diámetro equivalente del grano de oro

$$
\begin{aligned}
& \mathrm{a}=\text { largo } \\
& \mathrm{b}=\text { ancho } \\
& \mathrm{c}=\text { grosor }
\end{aligned}
$$

El índice de aplastamiento (IA) se determinó mediante la expresión:

$$
I A=\frac{\mathrm{c}}{\sqrt{\mathrm{a} \cdot \mathrm{b}}}
$$

A partir de los concentrados de oro de cada placer, se confeccionaron probetas pulidas para el análisis cuantitativo y cualitativo por microscopio electrónico de barrido con analizador de energías (SEM-EDS) y microsonda electrónica en los Serveis Científico-Tècnics de la Universitat de Barcelona. El SEM-EDS utilizado fue un equipo Cambridge Stereoscan-120. El analizador de energías acoplado es un EDS constituido por un cristal de $\mathrm{Si}(\mathrm{Li})$ con ventana de Be distribuido por LINK. Mediante este método se realizaron análisis químicos cualitativos de los elementos presentes en las muestras.

Los análisis cuantitativos se realizaron mediante una microsonda electrónica (CAMECA SX-50). Las condiciones de trabajo fueron las siguientes: tensión de aceleración de $25 \mathrm{keV}$ y una corriente de sonda de $20 \mathrm{nA}$ midiendo sobre las líneas espectrales $\mathrm{L} \alpha$ del $\mathrm{Au}$, con un cristal de $\mathrm{LiF}, \mathrm{L} \alpha$ de la Ag con un cristal PET, $\mathrm{K} \alpha$ del $\mathrm{Cu}$ con cristal $\mathrm{LiF}, \mathrm{K} \alpha$ del Fe con cristal LiF y $\mathrm{K} \alpha$ del Ni con cristal LiF. Los patrones utilizados fueron metales puros. 


\section{RESULTADOS}

\section{Características morfológicas de las partículas de oro en los placeres estudiados}

Las partículas de oro en las Playas Mejías y Jiguaní son muy finas, mostrando formas tabulares predominantemente; en ocasiones estos granos están muy aplastados formando laminillas u hojuelas, presentando contornos irregulares, sinuosos, cóncavos y convexos. Las dimensiones de las partículas son también variables, oscilando sus valores de largo entre $0,073 \mathrm{~mm}$ y $0,19 \mathrm{~mm}$, mientras que el ancho de las partículas osciló entre 0,036 y $0,10 \mathrm{~mm}$ y su grosor varió entre 0,005 a $0,018 \mathrm{~mm}$. El diámetro equivalente de los granos fluctuó entre 0,088 y $0,182 \mathrm{~mm}$ para ambos placeres (Cuadro 2). En resumen, podemos concluir que el oro presente en las arenas negras de los placeres estudiados es mucho más laminar que el aluvial de la región Sagua-Moa. Esta morfología laminar y muy aplastada del oro está en plena correspondencia con la dinámica costera y con los efectos mecánicos producidos por la combinación de las olas, las mareas y tormentas marinas predominantes en estos ambientes costeros.
Los valores promedios del índice de aplastamiento (IA) de los granos de oro en ambos placeres oscilaron entre 0,182 y 0,088 (Cuadro 2). Comparándolos con los obtenidos en las partículas de oro de los depósitos fluviales del río Quesigua (Fig. 3) apreciamos un notable decrecimiento de este parámetro morfométrico, indicando evidentemente un mayor transporte sufrido por las partículas reveladas en los placeres investigados. Las dimensiones máximas de las partículas de oro, en ambos placeres, no sobrepasaron los 0,196 mm.

El aplastamiento de los granos es mayor cuanto menor sea su IA (Giusti, 1986). Asumiéndose que a menor IA mayor es el transporte experimentado por las partículas, los valores de IA cercanos a la unidad corresponden a partículas prácticamente esféricas que han sufrido un menor transporte (Giusti, 1986). Así, el análisis del comportamiento del IA (Fig. 3) sugiere la existencia de fuentes de oro en posición distal a los depósitos de placeres marinos investigados, por lo que podemos señalar la relativa lejanía de la mineralización aurífera primaria suministradora del oro a los placeres Mejías y Jiguaní, respectivamente.

Cuadro 2

Promedios de mediciones tridimensionales y determinaciones morfométricas de las partículas de oro estudiadas en los placeres marinos del noreste de Cuba

\begin{tabular}{|c|c|c|c|c|c|c|c|}
\hline & \multirow[t]{2}{*}{ Muestra } & \multirow{2}{*}{$\begin{array}{c}\text { \# } \\
\text { partículas }\end{array}$} & \multicolumn{3}{|c|}{ Dimensiones de los granos (mm) } & \multicolumn{2}{|c|}{ Parámetros Morfométricos } \\
\hline & & & $\begin{array}{l}\text { Largo } \\
(\mathrm{mm})\end{array}$ & $\begin{array}{l}\text { Ancho } \\
(\mathrm{mm})\end{array}$ & $\begin{array}{l}\text { Grosor } \\
(\mathrm{mm})\end{array}$ & $\begin{array}{c}\mathrm{D}_{\mathrm{eq}} \\
(\mathrm{mm})\end{array}$ & $\begin{array}{c}\mathrm{IA} \\
(\mathrm{mm})\end{array}$ \\
\hline \multirow{7}{*}{$\begin{array}{l}\text { Playa } \\
\text { Mejías }\end{array}$} & MJV-1C & 5 & 0,1232 & 0,069 & 0,0092 & 0,0998 & 0,0998 \\
\hline & MJV-1D & 4 & 0,1065 & 0,0443 & 0,0053 & 0,0881 & 0,0881 \\
\hline & MJV-1E & 4 & 0,0735 & 0,0368 & 0,0086 & 0,1690 & 0,1690 \\
\hline & MJV-2A & 4 & 0,1549 & 0,0892 & 0,0188 & 0,1826 & 0,1826 \\
\hline & MJV-2B & 3 & 0,1276 & 0,0501 & 0,0082 & 0,1333 & 0,1333 \\
\hline & MJV-2C & 4 & 0,1021 & 0,056 & 0,0106 & 0,1412 & 0,1412 \\
\hline & MJV-2E & 5 & 0,1514 & 0,0791 & 0,0159 & 0,1477 & 0,1477 \\
\hline \multirow{5}{*}{$\begin{array}{l}\text { Playa } \\
\text { Jiguaní }\end{array}$} & MJV-2F & 3 & 0,1158 & 0,0759 & 0,0141 & 0,1511 & 0,1511 \\
\hline & PJ-3A & 9 & 0,1499 & 0,1047 & 0,0185 & 0,1477 & 0,1477 \\
\hline & PJ-3B & 7 & 0,1304 & 0,0743 & 0,0163 & 0,1762 & 0,1762 \\
\hline & PJ-4A & 6 & 0,1342 & 0,0462 & 0,0138 & 0,1753 & 0,1753 \\
\hline & PJ-4B & 6 & 0,196 & 0,0669 & 0,0114 & 0,0996 & 0,0996 \\
\hline
\end{tabular}




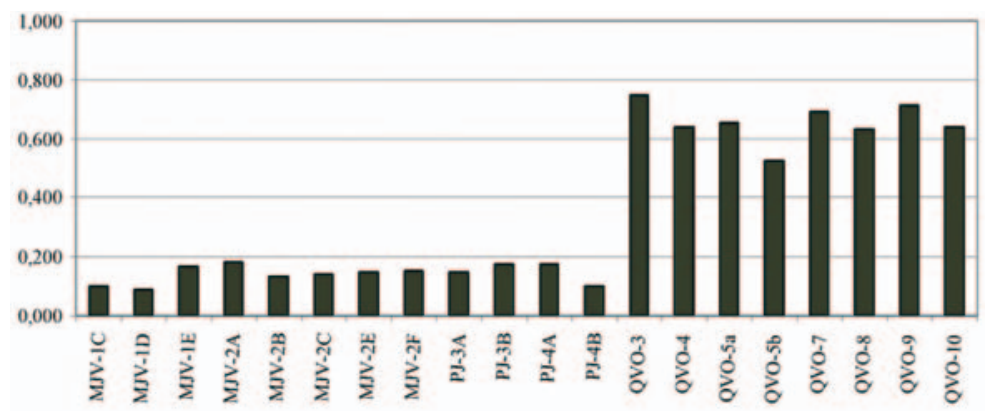

Fig. 3: Comportamiento del índice de aplastamiento (IA) de las partículas de Au en los depósitos de placeres marinos costeros de las playas Mejías (MJV) y Jiguaní (PJ). A manera de comparación también se muestra el IA de los granos de oro los depósitos fluviales del río Quesigua (QVO).

\section{Características texturales y composicionales de las partículas de oro}

Bajo el microscopio binocular las texturas externas de los granos de oro son predominantemente rugosas y porosas con claros efectos de corrosión, producidos posiblemente por procesos de disolución química. Texturas similares han sido observadas en otras regiones del mundo (Claudio et al., 1996). En ocasiones se observan superficies estriadas y lisas.

La observación de los rasgos morfológicos y texturales internos de las partículas de oro se realizó mediante el empleo de imágenes de electrones retrodispersados (Fig. 4) a partir de superficies pulidas, las cuáles ofrecen una información muy diferente a la obtenida mediante el empleo de un microscopio de polarización o lupa binocular.

Fig. 4: Imágenes de electrones retrodispersados de partículas de oro de composición compleja de los placeres Mejías (ABC) y Jiguaní (D). Nótese el aspecto corroído de los bordes y superficies de las partículas, así como granos de oro de diferentes composiciones. Las zonas más claras corresponden a las mayores concentraciones de $\mathrm{Au}$.
Las imágenes de electrones retrodispersados revelan la existencia de partículas de oro compuestas por subgranos de variadas formas, texturas y composición química. Los subgranos presentan texturas de corrosión con bordes sinuosos, cóncavos y convexos y muy cariados o alterados (Fig. 4D).

Los subgranos son de composición variable y algunos de ellos presentan composiciones correspondientes a electrum (Cuadro 3), mientras que otros son de composiciones próximas a las extremas, oro o de plata. Algunos subgranos de electrum se encuentran reemplazados por una generación de electrum más pobre en oro; otros, no. El contacto entre ambos tipos de electrum es con frecuencia difuso.
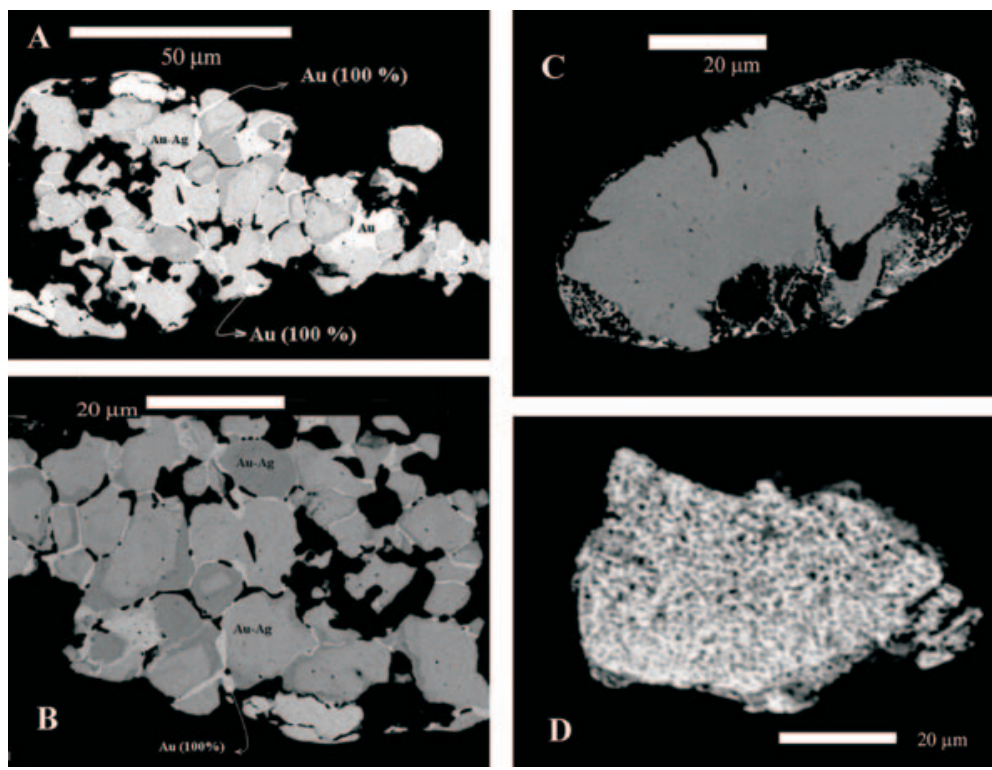
Cuadro 3

Resultados de los análisis químicos realizados por microsonda electrónica a los granos de oro de los placeres Mejías y Jiguaní

Placer Mejías (muestra MJV)

\begin{tabular}{lrrrrc}
\hline $\begin{array}{l}\mathrm{Au} \\
(\% \mathrm{w})\end{array}$ & $\begin{array}{c}\mathrm{Ag} \\
(\% \mathrm{w})\end{array}$ & $\begin{array}{c}\mathrm{Hg} \\
(\% \mathrm{w})\end{array}$ & $\begin{array}{c}\mathrm{Cu} \\
(\% \mathrm{w})\end{array}$ & $\begin{array}{c}\mathrm{Fe} \\
(\% \mathrm{w})\end{array}$ & $\begin{array}{c}\text { Total } \\
(\% \mathrm{w})\end{array}$ \\
\hline 61,85 & 34,34 & 3,48 & 0,14 & 0,01 & 99,82 \\
62,15 & 33,97 & 3,64 & 0,13 & 0,00 & 99,89 \\
95,85 & 3,95 & 0,25 & 0,08 & 0,00 & 100 \\
91,57 & 5,56 & 0,00 & 0,00 & 0,04 & 97,16 \\
64,39 & 33,97 & 2,76 & 0,09 & 0,00 & 100 \\
62,06 & 33,34 & 3,80 & 0,10 & 0,03 & 99,34 \\
61,80 & 34,63 & 3,27 & 0,28 & 0,01 & 99,99 \\
62,01 & 35,23 & 3,35 & 0,17 & 0,00 & 100 \\
74,20 & 24,77 & 0,00 & 0,03 & 0,05 & 99,04 \\
74,62 & 25,07 & 0,25 & 0,01 & 0,04 & 99,99 \\
97,86 & 2,45 & 0,00 & 0,01 & 0,00 & 100 \\
98,14 & 1,55 & 0,00 & 0,04 & 0,09 & 99,81 \\
94,48 & 2,12 & 0,04 & 0,02 & 0,20 & 96,85 \\
95,02 & 5,64 & 0,00 & 0,01 & 0,04 & 100 \\
96,63 & 3,07 & 0,00 & 0,01 & 0,09 & 99,80 \\
53,19 & 46,23 & 0,00 & 0,04 & 0,01 & 99,46 \\
54,22 & 44,50 & 0,08 & 0,02 & 0,02 & 98,83 \\
74,25 & 25,12 & 0,10 & 0,01 & 0,03 & 99,60 \\
74,59 & 24,62 & 0,22 & 0,03 & 0,01 & 99,47 \\
92,48 & 6,66 & 0,01 & 0,03 & 0,04 & 99,21 \\
64,03 & 38,28 & 0,00 & 0,03 & 0,00 & 100 \\
66,64 & 35,62 & 0,08 & 0,05 & 0,01 & 100 \\
69,23 & 31,78 & 0,00 & 0,03 & 0,02 & 100 \\
97,45 & 2,96 & 0,00 & 0,04 & 0,01 & 100 \\
73,30 & 25,39 & 0,06 & 0,00 & 0,01 & 98,76 \\
73,44 & 26,32 & 0,18 & 0,06 & 0,04 & 100 \\
29,26 & 51,49 & 18,76 & 0,05 & 0,02 & 99,58 \\
29,20 & 50,44 & 18,02 & 0,02 & 0,01 & 97,69 \\
28,47 & 49,96 & 20,12 & 0,00 & 0,00 & 98,56 \\
28,59 & 49,65 & 19,30 & 0,04 & 0,00 & 97,57 \\
28,17 & 50,47 & 19,28 & 0,00 & 0,00 & 97,93 \\
27,37 & 50,01 & 19,30 & 0,00 & 0,00 & 96,67 \\
28,85 & 50,65 & 18,79 & 0,04 & 0,00 & 98,33 \\
28,69 & 51,90 & 17,86 & 0,04 & 0,05 & 98,53 \\
\hline & & & & & \\
\hline
\end{tabular}

Placer Jiguaní (muestra PJ)

\begin{tabular}{lccccc}
\hline $\begin{array}{l}\mathrm{Au} \\
(\% \mathrm{w})\end{array}$ & $\begin{array}{c}\mathrm{Ag} \\
(\% \mathrm{w})\end{array}$ & $\begin{array}{c}\mathrm{Hg} \\
(\% \mathrm{w})\end{array}$ & $\begin{array}{c}\mathrm{Cu} \\
(\% \mathrm{w})\end{array}$ & $\begin{array}{c}\mathrm{Fe} \\
(\% \mathrm{w})\end{array}$ & $\begin{array}{c}\text { Total } \\
(\% \mathrm{w})\end{array}$ \\
\hline 94,32 & 0,33 & 0,22 & 2,53 & 0,38 & 97,78 \\
100,23 & 0,08 & 0,00 & 0,03 & 0,04 & 100 \\
92,81 & 0,12 & 0,15 & 2,91 & 0,20 & 96,18 \\
86,40 & 0,00 & 0,00 & 3,95 & 0,17 & 90,52 \\
92,94 & 0,00 & 0,00 & 2,67 & 0,18 & 95,79 \\
92,33 & 0,09 & 0,00 & 3,42 & 0,11 & 95,95 \\
89,73 & 0,17 & 0,00 & 3,16 & 0,03 & 93,09 \\
92,25 & 0,00 & 0,46 & 2,90 & 0,28 & 95,89 \\
86,40 & 0,00 & 0,00 & 3,37 & 0,31 & 90,08 \\
93,08 & 0,00 & 0,00 & 3,18 & 0,15 & 96,40 \\
\hline
\end{tabular}

Se trata de pepitas compuestas por subgranos de composición diversa, alterados en sus bordes y empobrecidos en oro en relación a la plata, y finalmente cementados por una generación tardía de oro puro (Fig. 4 A, B). Las partículas se encuentran corroídas de forma variable (Fig. 4 C).

La generación tardía de oro, en forma de películas intergranulares o vetillas de contactos muy netos (Fig. 4 A, B), se encuentra dispuesta según los bordes de los granos o penetrando dentro de los mismos a partir de los contactos entre los subgranos. A menudo esta generación de oro corta a la generación de electrum más pobre en oro.

El análisis composicional a nivel de subgrano se refleja en los diagramas ternarios $\mathrm{Au}$ $\mathrm{Ag}-\mathrm{Hg}$ y $\mathrm{Au}-\mathrm{Ag}-\mathrm{Cu}$. Según el diagrama ternario $\mathrm{Au}-\mathrm{Ag}-\mathrm{Hg}$ (Fig. 5B) el oro del placer Mejías muestra un espectro composicional más variado que el oro de Jiguaní, con composiciones a nivel de subgranos, correspondientes a electrum, mientras que otros son de composiciones próximas al oro nativo y otros con elevados valores de mercurio, hasta un $20 \%$ en peso aproximadamente.

El oro de la playa Jiguaní es mucho más noble desde el punto de vista composicional, con valores de plata que varían entre 20-42\% en peso y de oro entre $60-80 \%$ en peso lo que corresponde con valores próximos al electrum. También se presentan partículas de oro nativo, con casi el $100 \%$ de Au. El mercurio en ambas playas es anómalo aunque en Mejías se alcanzan valores de hasta un $20 \%$ en peso, lo que pudiera indicar la presencia de mineralización de oro mercurial, pudiendo asociarse con la existencia de depósitos epitermales, reportados en la región Sagua-Moa (Ramayo, 2003), donde el mercurio está presente.

$\mathrm{El}$ diagrama ternario $\mathrm{Au}-\mathrm{Ag}-\mathrm{Cu}$ (Fig. 5A) de las partículas de oro en los placeres investigados, refleja las variaciones composicionales existentes entre ambos depósitos. Los contenidos puntuales de cobre en las partículas de oro del placer Jiguaní, a nivel de subgranos, oscilan entre 5 y $15 \%$ en peso, lo que evidencia la existencia de subgranos con aleaciones intermetálicas de $\mathrm{Au}-\mathrm{Cu}$, muy similares al tetraauricúprido, el cual no ha sido reportado hasta el momento en nuestro país. Esta composición química de las 


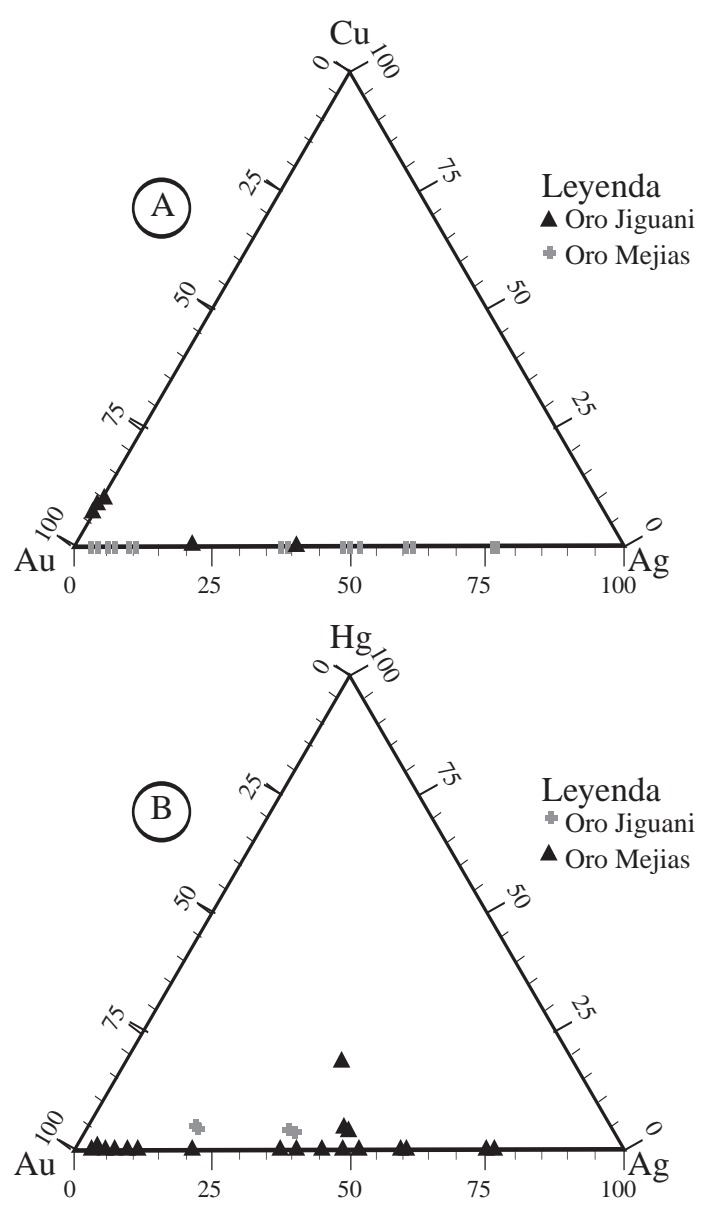

Fig. 5: Composición química de las partículas de oro estudiadas en los sistemas $\mathrm{Au}-\mathrm{Ag}-\mathrm{Cu}$ (A) y $\mathrm{Au}-\mathrm{Ag}-\mathrm{Hg}$ (B).

partículas de oro $(\mathrm{Au}-\mathrm{Cu})$, es propia de ambientes con un amplio desarrollo de rocas ultrabásicas (principalmente serpentinitas), las cuáles se encuentran en toda la cuenca del río Jiguaní (principal fuente suministradora de material detrítico al placer lateral de la playa Jiguaní), mientras que los granos de oro del placer Mejías prácticamente no contienen cobre, por lo que podemos establecer que hacia la porción oriental de la región de estudio existen fuentes vinculadas directamente con el desarrollo de rocas de afinidad ofiolítica (rocas ultramáficas), que aportan el oro, las cuáles no están presentes en el distrito Sagua de Tánamo (Fig. 2).

\section{DISCUSIÓN Y CONCLUSIONES}

La existencia de subgranos de diferente composición en una misma partícula (Fig. 4A) y la presencia de partículas de oro con bordes en los que el contenido de este metal es mayor respecto al resto de la partícula (Fig. 4C) son las características texturales más importantes de los granos de oro analizados en el placer Mejías. Estas características no pueden explicarse como resultado de un simple proceso de acreción química (Mann, 1984; Clough et al., 1989; Díaz-Martínez et al., 1998, 2002). En cambio, sugieren un proceso multiepisódico de formación de las partículas: (1) lixiviación de oro de los minerales primarios, (2) coalescencia de partículas de oro, y (3) enriquecimiento supergénico del oro en las partículas. Por otra parte, en las partículas investigadas en el placer Jiguaní, sobresalen texturas de corrosión con bordes sinuosos, cóncavos y convexos y muy cariados o alterados (Fig. 4D)

La existencia de subgranos de diferente composición dentro de una misma partícula es más coherente con un origen a partir de un proceso de acreción mecánica de partículas de oro, favorecidas por la maleabilidad de este metal. Las partículas de oro presentes en los depósitos del placer Mejías incluyen subgranos que registran historias geológicas (fuentes primarias, transporte, lixiviación selectiva, enriquecimiento supergénico) diferentes. Las partículas actuales son el resultado de la acreción mecánica de estos subgranos en el medio fluvial. Eyles \& Kocsis (1989) también invocan un mecanismo de acreción mecánica para explicar la formación de granos mayores en los placeres, dado que el tamaño de los granos de oro en los placeres suele ser mucho mayor que el encontrado en las fuentes primarias. Por otra parte, las superficies de separación de los subgranos han actuado como superficies de debilidad estructural, favoreciendo la alteración química de las partículas.

A diferencia de otros depósitos estudiados en la región (Díaz-Martínez et al., 1998, 2002), las partículas de oro analizadas en ambos placeres presentan contenidos anómalos de $\mathrm{Hg}$, alcanzando en algunas partículas hasta un $20 \%$ en peso aproximadamente, lo que pudiera indicar la 
presencia de mineralización de oro mercurial, pudiendo asociarse con la existencia de depósitos epitermales, reportados en la región SaguaMoa (Ramayo, 2003), donde el mercurio está presente; por otra parte las partículas de oro reveladas en el placer Jiguaní contienen entre un 5 y un $15 \%$ de $\mathrm{Cu}$, lo que evidencia la presencia de partículas auríferas compuestas por aleaciones intermetálicas de $\mathrm{Au}-\mathrm{Cu}$, muy similares al tetraauricúprido, el cuál no ha sido reportado hasta el momento en nuestro país.

Sí comparamos estos contenidos de $\mathrm{Cu}$, con los determinados en las partículas auríferas de los depósitos fluviales del río Cayo Guam (varían entre 4,75 y $55 \% \mathrm{Cu}$ ), podemos constatar que las partículas extraídas de las arenas grises del placer Jiguaní, presentan contenidos más bajos de este metal, condicionado al parecer por los procesos de lixiviación marina, provocados por ataque químico o autoelectrorefinación (Claudio et al., 1996) a que son sometidos las partículas de $\mathrm{Au}$ en este placer y que es función directa del tiempo de permanencia del oro en estos depósitos marinos costeros.

La composición química Au-Ag (electrum) y oro nativo $(\mathrm{Au})$ de las partículas auríferas puede estar ligada principalmente a la presencia de filones y brechas hidrotermales enriquecidas en oro en la región Sagua-Moa (Ramayo, 2003). Según este autor, las fases minerales portadoras de oro en estos filones presentan formas euedrales y subedrales, y poseen diámetros que varían desde 20 a $55 \mu \mathrm{m}$. Los estudios realizados por microsonda electrónica sobre estos granos de oro, arrojaron términos composicionales ricos en Au con valores superiores al $86 \%$ en peso, no observándose significativas variaciones composicionales. Estos datos sugieren señalar a los indicios hidrotermales investigados por Ramayo (2003) como una de las principales fuentes primarias de suministro a los depósitos de placeres investigados. Sin embargo, no se puede descartar el aporte de otras posibles fuentes primarias, ya que en la región objeto de estudio existen diversos metalotectos para oro: rocas ofiolíticas, listvenitas, lateritas. Por ejemplo, Proenza (1997) encuentra granos de oro libre en las cromititas de la faja Mayarí-Baracoa. Estos granos son de dimensiones muy pequeña (entre 5 y 10 micras), en estas partículas se da una amplia variación composicional en cuanto a la relación $\mathrm{Au} / \mathrm{Ag}$, de forma análoga a lo que acontece en los depósitos marinos costeros investigados.

\section{AGRADECIMIENTOS}

El presente trabajo forma parte de la tesis doctoral del primer autor. Los análisis de SEMEDS y de microsonda electrónica fueron realizados en los Serveis Científico-Tècnics de la Universitat de Barcelona. Especialmente los autores quieren agradecer la ayuda del Dr. X. Llovet durante los análisis de microsonda. Este trabajo es una contribución a los proyectos BTE2001-3308 y a un proyecto de la AECI (Programa de Cooperación Científica con Iberoamérica 2000).

\section{REFERENCIAS}

ADAMOVICH, A. \& CHEJOVICH, V.D., 1964: Principales características de la geología y de los minerales útiles de la región nordeste de la provincia de Oriente. - Rev. Tecnológica, 2: 14-20.

ANDÓ, J., KOZÁK, M. \& RÍOS, Y., 1989: Caracterización general de la asociación ofiolítica de la zona Holguín-Moa y el papel de las ofiolitas en el desarrollo estructural de Cuba. - Rev. Minería y Geología, 1: $15-33$.

CLAUDIO, G. \& MARTIN, H., 1996: Mineralogy, morphology, and chemistry of gold in the stone line lateritic profile of the Posse deposit, central Brazil. - J. Geochem. Exploration, 57(2): 115-125.

CLOUGH, D. M. \& CRAW, D., 1989: Authigenic gold-marcasite association: evidence for nugget growth by chemical accretion in fluvial gravels, Southland, New Zealand. - Economic Geol. 84: 953-958.

DÍAZ-MARTÍNEZ, R., 1995, Sobre la existencia de placeres lateral en el Noreste de Cuba Oriental. - Rev. Minería y Geología, 12: 39-41.

DÍAZ-MARTÍNEZ, R, 1996: Distribución del oro, platinoides y otros minerales en los placeres de la costa norte de Cuba Oriental. - 112 págs. Inst. Sup. Minero Metalúrgico, Moa, Holguín, Cuba [Tesis Ph.D.]. 
DÍAZ-MARTÍNEZ, R., PROENZA, J.A., COMAS, J., FERNÁNDEZ-BELLON, O., FABRA, J.M., GUINART, O. \& MELGAREJO, J.C., 1998: El placer lateral de playa Mejías (noreste de Cuba Oriental): un ejemplo de interacción de procesos aluviales y marinos en la concentración de minerales de elementos preciosos. - Acta Geol. Hispanica, 33: 351-371.

DÍAZ-MARTÍNEZ, R., MELGAREJO, J.C., PROENZA, J.A. \& VILA, A.R., 2002: Texturas y composición de partículas de oro asociadas a depósitos de placeres en el NE de Cuba: evidencias para un debate sobre lixiviación y/o acreción mecánica. - Bol. Soc. Española Mineralogía, 25-A: 27-28.

EYLES, N. \& KOCSIS, S.P., 1989: Sedimentological controls on gold in a late Pleistocene glacial placer, $\mathrm{Ca}$ riboo mining district, British Columbia, Canada. Sed. Geol. 65: 45-68.

FONSECA, E., ZELEPUGIN, V.N. \& HEREDIA, M., 1985: Structure of the ophiolite association of Cuba. - Geotectonic, 19: 321-329.

GARCÍA-TORRES, M.C., CARRALERO, N., PROENZA, J. \& BLANCO, J., 1996: Condiciones paleotectónicas y de sedimentación de las formaciones Sabaneta y Vigía según el modelo de una cuenca de retroarco. - Rev. Minería y Geología, 13: 13-17.

GIUSTI, L., 1986: The morphology, mineralogy, and behaviour of fine - grained gold from placer deposits of Alberta: Sampling and implication for mineral exploration. - Canadian J. Earth Sci. 23: 16621672.

ITURRALDE-VINENT, M. A, 1976: Estratigrafía de la zona Calabazas-Achotal, Mayarí Arriba. Oriente. - La Minería en Cuba, 5: 9-23

ITURRALDE-VINENT, M.A., 1994: Cuban Geology: a new plate-tectonic síntesis. - J. Petrol. Geol., 17(1): 39-70.

ITURRALDE-VINENT, M. A., 1996: Geología de las ofiolitas de Cuba. - En: ITURRALDE-VINENT, M. (ed.): Ofiolitas y arcos volcánicos de Cuba. - IGCP project 364, Special Contribution, 1: 83-120.

ITURRALDE-VINENT, M. A., 1996b. Cuba: el arco de islas volcánicas del Cretácico. - En: ITURRALDE-VINENT, M. (ed.): Ofiolitas y arcos volcánicos de Cuba. - IGCP project 364, Special contribution, 1: 179-189.

KERR, A.C., ITURRALDE-VINENT, M., SAUNDERS, A.D., BABBS, T.L. \& TARNEY, J., 1999: A new plate tectonic model of the Caribbean: Implications from a geochemical reconnaissance of Cuban Mesozoic volcanic rocks. - GSA Bull. 111: 1581-1599.

KULASKOV, L., DÍAZ-MARTÍNEZ, R. \& RODRÍGUEZVEGA, A., 1990: Reconocimiento y evaluación pronóstico sobre la concentración de minerales pesados en los placeres laterales en las cercanías del macizo ofiolítico Mayarí-Baracoa. [Informe interno]. 35 págs. Fondo Geol. ISMM Moa.

MANN, A.W., 1984: Mineralogy, morphology and chemistry of gold in the stone line lateritic profile of the Posse deposit. - Economic Geol. 79: 8-49.

MILLÁN, G., 1996: Metamorfitas de la asociación ofiolítica de Cuba. - En: ITURRALDE-VINENT, M. (ed.): Ofiolitas y arcos volcánicos de Cuba. - IGCP Project 364, Special Contribution, 1: 83-120.

NAGY, E., BREZSNYÁNSZKY, K., BRITO, A., COUTIN, D., FORMELL, F., FRANCO, G., GYARMATTÍ, P., RADOCZ, G. \& JAKUS, P., 1983: Contribución a la geología de Cuba Oriental. - 273 págs. Ed. Científico-Técnica, La Habana.

PROENZA, J. \& CARRALERO, N., 1994: Un nuevo enfoque sobre la geología de la parte sur de la cuenca de Sagua de Tánamo. - Rev. Minería y Geología, 11: 3-10.

PROENZA, J.A., 1997: Mineralización de cromita en la faja ofiolítica Mayarí-Baracoa (Cuba): Ejemplo del yacimiento Mercedita. - 227 págs. Univ. de Barcelona, España, [Tesis Ph.D.].

PROENZA, J., MELGAREJO, J.C., GERVILLA, F., LAVAUT, W., REVÉ, D. \& RODRÍGUEZ, G., 1998: Cromititas podiformes en la Faja Ofiolítica Mayarí-Baracoa (Cuba). - En: Melgarejo, J. C. \& Proenza, J.A. (eds.): Geología y metalogenia de Cuba: Una introducción. - Acta Geol. Hispánica, 33(1-4): 153-177.

PROENZA, J., GERVILLA, F., MELGAREJO, J.C. \& BODINIER, J.L. 1999a: Al- and Cr- rich chromitites from the Mayarí-Baracoa Ophiolitic Belt (eastern Cuba): consequence of interaction between volatilerich melts and peridotite in suprasubduction mantle. - Economic Geol. 94: 547-566.

PROENZA, J., GERVILlA, F. \& MELGAREJO, J.C. 1999b: La Moho Transition Zone en el Macizo Ofiolítico Moa-Baracoa: un ejemplo de interacción magma/peridotito. - Rev. Soc. Geol. España 12(34): $309-327$. 
QUINTAS-CABALLERO, F., 1989: Análisis estratigráfico y paleogeografía del Cretácico Superior y del Paleógeno de la provincia Guantánamo y áreas cercanas. 161 págs. Inst. Sup. Minero Metalúrgico, Moa, Holguín, [Tesis Ph.D.].

QUINTAS, F \& BLANCO., J: 1993: Paleogeografía de la cuenca San Luis y su importancia para la interpretación de la evolución geológica de Cuba Oriental. Rev. Minería y Geología, 10: 3-14.

QUINTAS, F., 1996, Bioestratigrafía del corte Meso - Cenozoico del extremo oriental de Cuba. - Rev. Minería y Geología, 13(1): 3-8.
RAMAYO-CORTÉS, L., 2003: Geoquímica y mineralogía de la mineralización epitermal de la región nororiental de la provincia Holguín. Ejemplos de las manifestaciones minerales Hato Viejo y El Rifle-Los Calderos. - 127 págs. Inst. Sup. Minero Metalúrgico Moa, Holguín, [Tesis Ph.D ]

SAWKINS, F. J., 1990: Metal deposits in relation to plate tectonics. -460 págs. Springer, Berlin.

VILA, A., 1999, Principales rasgos de la distribución del oro en los depósitos exógenos de la región Sagua-Moa. 80 págs. Inst. Sup. Minero Metalúrgico, Moa, Holguín, [Tesis McS.]. 